\title{
PASARELA PEATONAL ATIRANTADA EN LA CARRETERA DE ACCESO A LA UNIVERSIDAD DE VIGO
}

\author{
(STAYED FOOT-BRIDGE IN VIGO CROSSES THE MAIN ACCESS TO THE UNIVERSITY)
}

\author{
Antonio González Serrano, Ingeniero de Caminos \\ Julio Besiga Díaz-Blanco, Arquitecto \\ Ricardo López Escapa, Ingeniero de caminos \\ Fernando Blanco Ibáñez, Jefe de Obra
}

ESPAÑA
Fecha de recepción: 30-IV-99

$562-154$

\section{RESUMEN}

En este artículo se expone una pasarela peatonal atirantada, situada en la carretera de acceso a la Universidad de Vigo. La pasarela tiene una planta en forma de $U$, estando atirantada en su borde interior, formado por un cajón metálico, del que se suspende y en el que se empotra el tablero, constituido por una estructura mixta nervada, de canto variable, muy esbelta.

\section{SUMMARY}

This paper dealts with the design and the construction of a stayed foot-bridge in Vigo, crosses the main access to the university. The foot-bridge have the shape in plant of one $U$, suspended from four sets of ties, arranged along the inner side of the deck. The deck is built in a metalic box, wich is suspended from the ties. The cantilever of the deck with a variable depth results in a very light structure whose grace can be seen at a glance.

\section{Introducción}

Se describe la idea básica que dio origen al proyecto, la transformación de esa idea en una estructura coherente y sencilla y el proceso constructivo de una pasarela peatonal, atirantada en su borde interior, situada en la carretera de acceso a la Universidad de Vigo.

\section{La sencillez de una idea}

La pasarela surge, fundamentalmente, ante la necesidad de realizar un paso superior peatonal que sirva para cruzar la nueva carretera de acceso, que va desde el Parque de Castrelos al Parque Tecnológico, al Parque Botánico y a la Universidad de Vigo. La nueva carretera es la ampliación de una existente, aumentando la anchura de este vial, aproximadamente, al doble.
La proximidad de un núcleo de población y la existencia de un centro escolar, hizo necesario el plantearse, simultáneamente, la ubicación y el diseño de esta pasarela.

La pasarela se ubicó en las proximidades del centro escolar, diseñándola, en primer lugar, como un "reclamo" para los alumnos, mostrándoles su rampa de acceso e invitándoles a su utilización $\mathrm{y}$, en segundo lugar, adaptándola a la topografía del lugar.

La carretera tiene, en este punto, una pendiente cercana al $9 \%$, con lo cual, la disposición de las rampas de acceso, en contra de la pendiente, permitió diseñarTas con un desarrollo reducido, lo que favorece consīerablemente su utilización, máxime teniendo en cuenta que los recorridos son relativamente cortos. 
La pasarela se diseñó en base a una planta en forma de U, con dos rampas paralelas, que forman los brazos de la $\mathrm{U}$, que, con una longitud de 26,50 metros, alcanzan el gálibo previsto, con una pendiente del 10 por ciento.

El tramo central de la pasarela es de 27 metros de longitud, habiéndose diseñado con un alzado en forma de V invertida, con un gálibo de 6,20 metros en el eje de la carretera, hecho que permite el paso de cualquier tipo de carga.

Se diseñaron dos pilas inclinadas que enmarcan el tramo central de la pasarela, en las que se anclan los tirantes, que están contenidos en tres planos verticales y que potencian espacialmente el recorrido.

El diseño de los elementos más singulares : el tablero, el nudo superior de la pila, los pilares de la rampa, las esquinas del tablero, las protecciones de los conectores en los tirantes de compensación, las proteccciones antivandálicas y la barandilla han sido tratados con el mayor cuidado y la mayor sencillez.

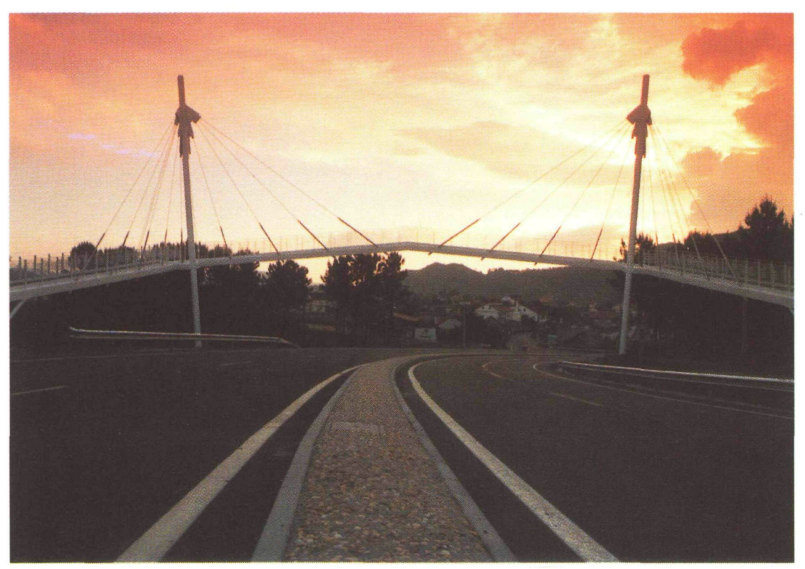

Foto 1.- Alzado que muestra el tramo central de la pasarela. Fotografia tomada desde la parte cóncava de la $U$.

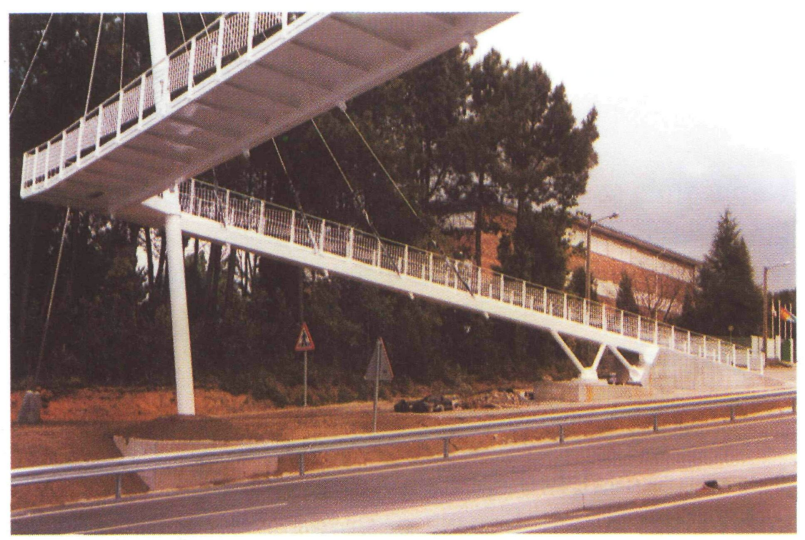

Foto 2.- Vista general de la pasarela, mostrando el otro lado de la misma. La fotografia se tomó desde la parte convexa de la $U$.

\section{Descripción de la estructura}

La pasarela está atirantada, únicamente, en su borde interior, mediante 18 tirantes, formados por cordones de 0,6", compuestos por 7 alambres, galvanizados y protegidos con cera petrolera, en el interior de una vaina de polietileno blanca resistente a la acción de los rayos solares.

La pasarela tiene un ancho total de 1,70 metros y un ancho útil de 1,50 metros. La sección transversal está compuesta por un cajón metálico de $300 \mathrm{~mm}$ de canto, $200 \mathrm{~mm}$ de ancho y $16 \mathrm{~mm}$ de espesor, que se diseñó para resistir las torsiones necesarias, además de los esfuerzos de flexión y el cortante. La suspensión del tablero con los tirantes se realiza puntualmente, en el eje del cajón.

En el cajón metálico se empotra un voladizo de 1,50 metros y que está formado por $1 / 2$ IPE de 200, dispuestos cada metro, cortados mediante un plano diagonal, de forma que presenten un canto linealmente variable, desde $150 \mathrm{~mm}$, en la zona de empotramiento en el cajón, hasta $50 \mathrm{~mm}$, en el borde libre.

A los perfiles, así cortados, se les soldó, por la parte superior, una chapa de $3 \mathrm{~mm}$ de espesor, provista de conectores, los cuales estaban formados por angulares de $40 \times 40 \times 4$, de $35 \mathrm{~mm}$ de longitud, dispuestos en retícula de $375 \times 3,5 \mathrm{~mm}$. Además de los conectores se dispuso un mallazo, formado por redondos de $12 \mathrm{~mm}$ de diámetro, que se soldó a los conectores.

A la chapa de $3 \mathrm{~mm}$ se le incorporó un angular corrido de 60x60x6, en el borde del cajón y un perfil UPN 100, también corrido, en el extremo del voladizo. De esta forma se crea una capa de micro hormigón, de 4,7 mm de espesor en el extremo del voladizo y de $6 \mathrm{~mm}$ de espesor en el empotramiento con el cajón, consiguiendo una buena utlización por parte del usuario, evitando el efecto tambor y la formación de heladas en el pavimento.

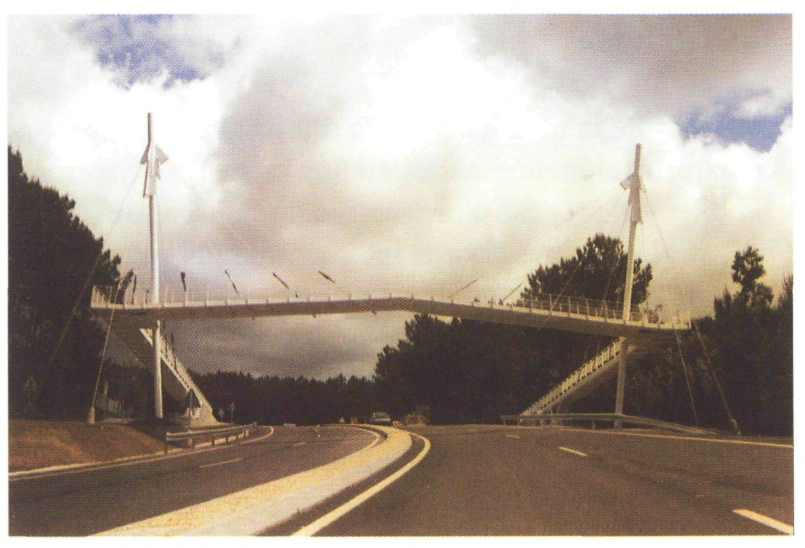

Foto 3.- Perspectiva del tablero, donde se v̄e el cajón de borde, los nervios de rigidez de la ménsula, la chapa de $3 \mathrm{~mm}$, que soporta la losa de micro hormigón, los tirantes de suspensión, una rampa de acceso y los tirantes de compensación.

http://informesdelaconstruccion.revistas.csic.es 
Los tirantes se suspenden de dos pilas tubulares metálicas de $36,8 \mathrm{~mm}$ de diámetro y $20 \mathrm{~mm}$ de espesor, que se diseñaron con una altura total de 16,50 metros. El cuelgue de los tirantes se realiza a 15,00 metros de altura. Ambas pilas se diseñaron inclinadas, unidas excéntricamente al tablero, de forma que los tirantes están contenidos en tres planos verticales que contienen a los ejes de los tubos de torsión del tablero.

El cajón metálico de las rampas de acceso está suspendido por cuatro tirantes, separados tres metros entre sí, estando apoyado, además, en tres pilares extremos, en forma de zig-zag. Uno de estos pilares parte del estribo y dos de los pilares apuntan al punto de suspensión de los tirantes en las pilas. Los pilares están formados por tubos de $177,8 \mathrm{~mm}$ de diámetro.

El cajón metálico del vano central está suspendido de ocho tirantes, separados, también, cada tres metros.

Además de los tirantes descritos, se diseñaron dos tirantes verticales que van desde las pilas a las intersecciones de los cajones metálicos que forman las rampas de acceso con el vano central.

También se diseñaron dos tirantes de compensación en cada pila, que van anclados en dos imuertos de hormigón. Los tirantes de compensación de la rampa están formados por un único cordón de 0,6 “, mientras que los tirantes de compensación del tramo central están formados por dos cordones de 0,6 ".

Los anclajes de todos los tirantes en la pila están formados por manguitos de extrusión, mientras que los anclajes de todos los tirantes en la cara inferior del tablero son anclajes activos.

Los anclajes de los tirantes de compensación están formados por conectores, que se proyectaron embebidos

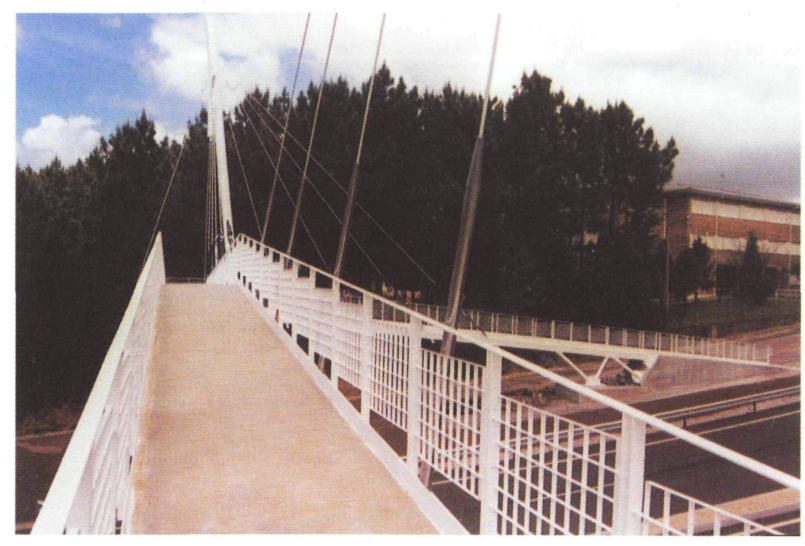

Foto 4.- Vista superior del tablero, los tirantes, una pila y una rampa de acceso.

(c) Consejo Superior de Investigaciones Científicas Licencia Creative Commons 3.0 España (by-nc) en un cono de hormigón armado que sobresale de los muertos de anclaje, para su protección futura.

La pila se diseñó empotrada en la zapata. Primeramente se construyó la zapata, en la que se realizó un aligeramiento cilíndrico en su interior, delimitado por un tubo metálico, debidamente anclado al hormigón de la zapata, de mayor diámetro que el de la pila. Durante la fase de montaje, la pila se enfiló y se conectó con el tubo del aligeramiento, retacando la unión con un mortero de altas prestaciones mecánicas.

La cimentación de la pasarela es una cimentación directa convencional. Las zapatas se diseñaron para una tensión admisible de $0,15 \mathrm{~N} / \mathrm{mm}^{2}$. Los muertos de hormigón para el anclaje de los tirantes de compensación se diseñaron con los coeficientes de seguridad que establece la E.H. vigente.

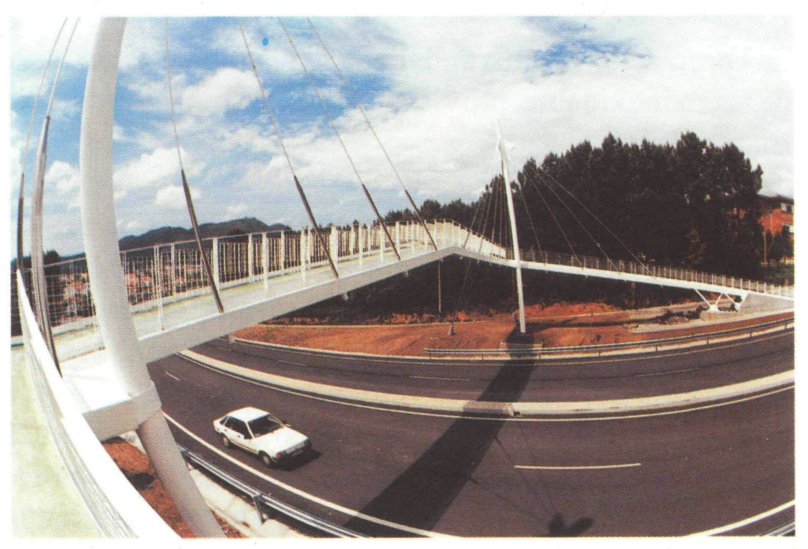

Foto 5.- Vista general sesgada de la pasarela.

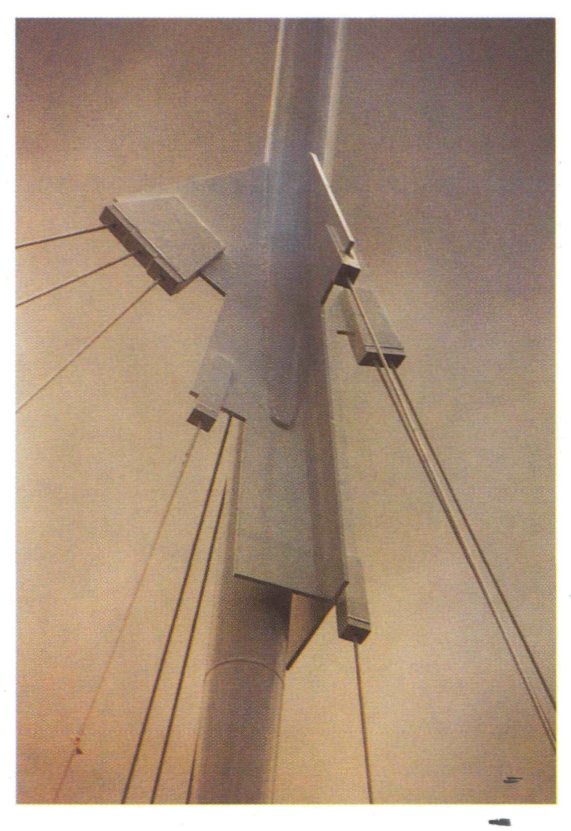

Foto 6.- Cabeza de la pila donde se anclan los tirantes. 


\section{Elementos singulares}

El diseño de los elementos más singulares : el tablero, el nudo superior de la pila, los pilares de la rampa, las esquinas del tablero, las protecciones de los conectores de los tirantes de compensación, las proteccciones antivandálicas y la barandilla, se han tratado -como se ha indicado- con la mayor sencillez.

La cabeza, o parte superior de la pila, que recibe a todos los tirantes, se ha diseñado intentando crear un elemento armónico, equilibrado y de notable belleza.

La función resistente de las esquinas de la pasarela se corresponde con una estructura funcional y estética.

El tablero se diseñó con una notable esbeltez, trabajando en voladizo, empotrado en un cajón, del que está suspendida la pasarela, con un canto de $100 \mathrm{~mm}$ en el borde libre.

Las protecciones antivandálicas se resolvieron con tubos de acero inoxidable, provistos de una terminación superior troncocónica.

La barandilla debía resolver una doble función : la seguridad, sin olvidar la estética. La sencillez de la pasarela se tenía que ver reflejada en este elemento, que no podía quitar protagonismo a la estructura. Se diseñó una barandilla formada por montantes verticales, enlazados, constituidos por una doble pletina, dispuestas de canto, de $5 \mathrm{~mm}$ de espesor, separadas entre sí $50 \mathrm{~mm}$. Entre los montantes se dispuso, a modo de pasamanos, una pletina, también de canto, de $10 \mathrm{~mm}$ de espesor. En el espacio delimitado por las pletinas, se colocó una pletina en retícula, de $100 \mathrm{~mm}$, bordeada por cuatro pletinas, dispuestas, también, de canto.

\section{Proceso de tesado}

Para tesar la pasarela se apuntaló la estructura en cuatro puntos, situando dos puntales en el tramo principal y un

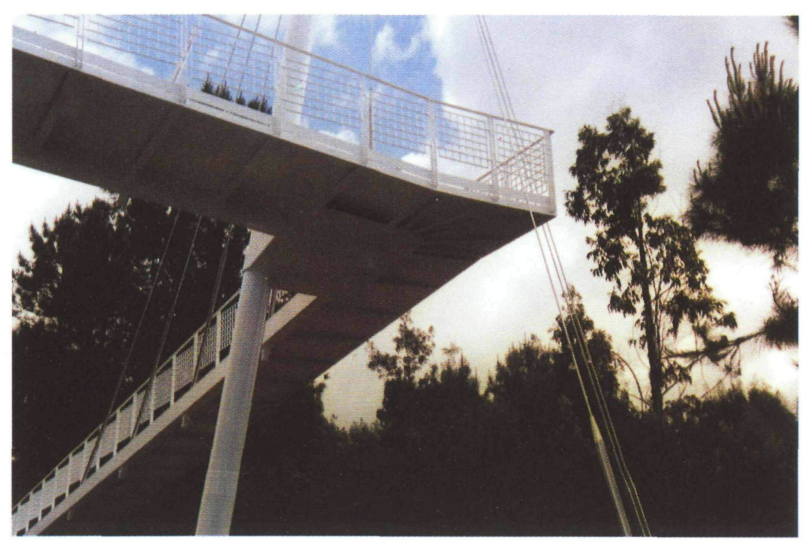

Foto 7.- Vista inferior del nudo de esquina del tablero.

(c) Consejo Superior de Investigaciones Científicas Licencia Creative Commons 3.0 España (by-nc) puntal en cada rampa de acceso. Se lastró la pasarela, al mismo tiempo, en estos puntos.

La puesta en tensión se hizo en dos escalones, de acuerdo con el plan establecido en el proyecto, quedando la pasarela suspendida, una vez retirados los lastres, sin apoyarse en los puntales.

\section{Datos de la obra}

Pasarela peatonal. En la carretera de acceso desde el Parque de Castrelos al Parque Tecnológico, Parque Botánico y Universidad de Vigo.

Propiedad. Consellería de Política Territorial, Obras Públicas y Vivienda. Xunta de Galicia.

Director de la obra. Ricardo López Escapa. Ingeniero de Caminos.

Constructor. Dragados y Construcciones, S.A.

Proyectista. Antonio González Serrano. Ingeniero de Caminos. Patólogo.

Arquitecto Colaborador en el Proyecto : Julio Besiga Díaz-Blanco.

Jefe de Obra. Fernando Blanco Ibáñez.

Sistema de Pretensado. CTT.

\section{Reconocimientos}

No se quiere terminar este artículo sin agradecer la confianza deparada por José Luis Manso Alonso; la buena labor desarrollada por el Jefe de Obra, Fernando Blanco Ibáñez, en el cuidado de los detalles, así como la perfección en el proceso de tesado realizado por José Escobar Martínez, de CTT.

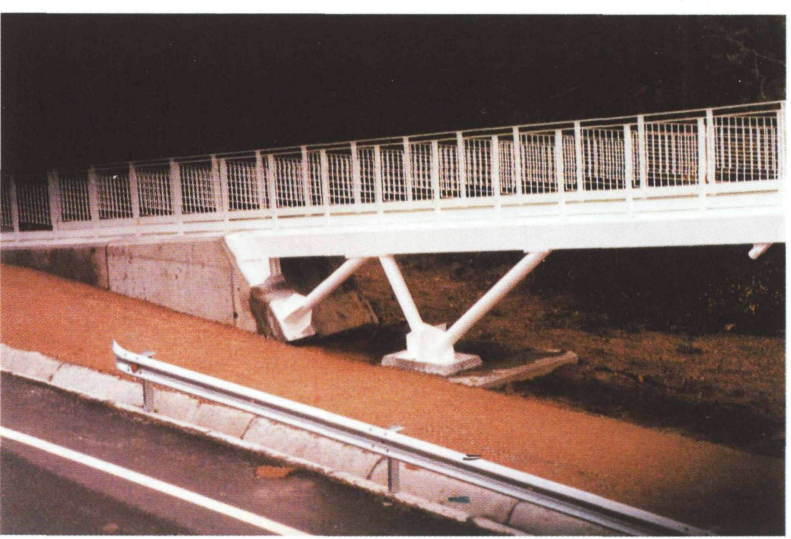

Foto 8. - Estribo y arranque de una rampa. http://informesdelaconstruccion.revistas.csic.es 